\title{
Seismic response characteristics of buried pipelines subjected to large ground movements during huge earthquakes
}

\author{
K. Fuchida \\ Department of Architecture and Civil Engineering, \\ National Institute of Technology, Kumamoto College, Japan
}

\begin{abstract}
This paper aims to investigate seismic response behavior of buried pipelines subjected to large ground displacements during huge earthquakes and evaluate the response characteristics of them for various conditions of ground and pipelines. The seismic response analysis method for surface ground layers uses 2-dimensional effective stress analysis based on Biot's two phase mixture theory and Iai's constitutive equation. The pipeline analysis is based on a beam theory on an elastic foundation and modified transfer matrix method. Numerical computations are performed for various models with changing conditions of ground and pipes. As a result, axial and lateral response characteristics of pipelines are shown and evaluated by relating with earthquakes, ground and pipelines conditions.

Keywords: response analysis, buried pipeline, surface layers, modified transfer matrix method.
\end{abstract}

\section{Introduction}

Since severe damages of buried pipelines (lifeline systems) during huge earthquakes affect the social activity and urban life of citizens greatly, it is important to investigate and reduce the responses of buried pipelines subjected to large scaled ground motions.

This research aims to propose an evaluating method for seismic responses of buried pipelines and study the response characteristics of buried pipelines which are subjected to large ground displacements with various conditions of pipelines and joints during earthquakes. 
The proposed method consists of two analytic methods. Firstly the responses of surface ground layers, in which a weak pipeline is buried, are investigated. Then detailed responses of pipelines are evaluated based on input soil displacements which are determined by referring to above responses of surface ground layers. The proposed method is briefly introduced as follows and some investigated examples of analyses are shown here.

\section{Evaluation method for pipeline responses}

The evaluating method for buried pipeline responses during earthquakes consists of two main analytic methods. The first method is the seismic response analysis for the surface ground layers. The analysis is performed by the existing computer program which is the effective stress analysis program "NUW2" [1, 2]. The program "NUW2" is based on Biot's two phase mixture theory and Iai's constitutive equation [3]. The second method is the pseudo static response analysis for the buried pipeline. This method is based on the beam theory on an elastic foundation and modified transfer matrix method [4-7]. The flow of this synthetic method is shown in Figure 1.

\subsection{Seismic response analysis for surface ground layers}

The analytic method for response of surface ground layers used in this study adopts the two-dimensional dynamic equilibrium equations for the soil-water phase and generalized Darcy law for the pore water based on Biot's two-phase mixture theory as in the references $[1,2]$. To treat non-linearity (liquefaction) of the soil in the near field, the constitutive model for the plain condition is introduced. This constitutive model is constructed based on the 2-D strain-space multimechanism model for cyclic mobility of sandy soil first proposed by Iai et $a l$. [3]. For the far field condition, the absorbing boundary condition is prepared.

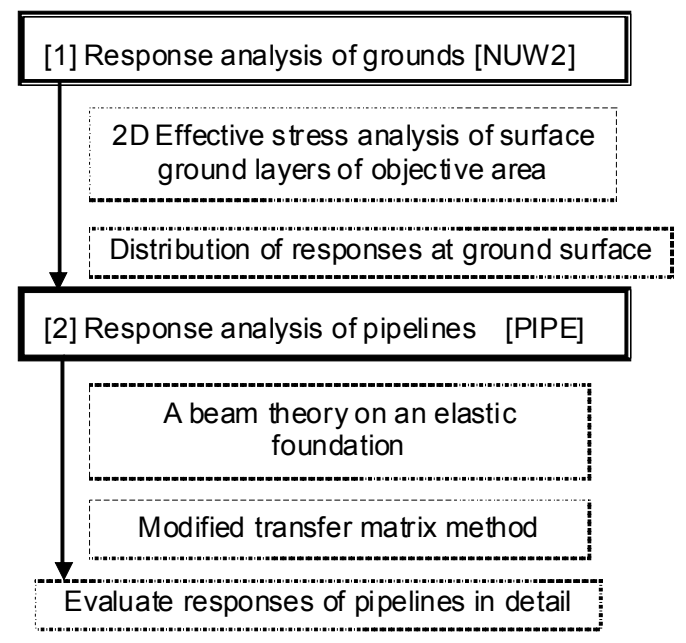

Figure 1: Flow of the evaluating method. 
The above dynamic equilibrium equations are formulated to the finite element equation by considering the irreducible weak Galerkin formulation. The matrix form of finite element equation for a saturated porous medium with compressible pore water including the absorbing boundary condition may be written as:

$$
\begin{aligned}
& \left(\begin{array}{ll}
m_{u u} & m_{u w} \\
m_{u w}^{T} & m_{w w}
\end{array}\right)\left\{\begin{array}{c}
\ddot{\bar{u}} \\
\ddot{\bar{w}}
\end{array}\right\}+\left(\begin{array}{cc}
0 & 0 \\
0 & c_{w w}
\end{array}\right)\left\{\begin{array}{c}
\dot{\bar{u}} \\
\dot{\bar{w}}
\end{array}\right\}+\left(\begin{array}{cc}
K_{u u} & K_{u w} \\
K_{u w}^{T} & K_{w w}
\end{array}\right)\left\{\begin{array}{l}
\bar{u} \\
\bar{w}
\end{array}\right\}+\left\{\begin{array}{c}
B_{\Omega} B_{u}^{T} \sigma^{\prime} d \Omega \\
0
\end{array}\right\} \\
& =\left\{\begin{array}{l}
\bar{f}_{u}+\bar{f}_{u}^{f} \\
\bar{f}_{w}+\bar{f}_{w}^{f}
\end{array}\right\}-\left(\begin{array}{cc}
c_{u u}^{\prime} & c_{u w}^{\prime} \\
c_{u w}^{\prime T} & c_{w w}^{\prime}
\end{array}\right)\left\{\begin{array}{c}
\dot{\bar{u}}-\dot{\bar{u}}^{f} \\
\dot{\bar{w}}-\dot{\bar{w}}^{f}
\end{array}\right\}
\end{aligned}
$$

where $\mathrm{u}$ and $\mathrm{w}$ are the nodal displacement vectors and other notations can be seen in the reference [2]. Equation (1) is coded as the 2D-FE effective stress analysis program of the name "NUW2".

\subsection{Response analysis for pipelines}

The responses of pipelines subjected to the ground displacement induced by liquefaction or seismic wave are analyzed by using the program "PIPE" [4-7], which is based on the beam theory on an elastic foundation. Replacing the stiffness of liquefied or non-liquefied ground around the pipeline with a coefficient of subgrade reaction, the pipeline is modeled as pipeline-soil spring system as shown in Figure 2. The pipeline segments are connected by the axial and rotational joint spring $k_{\mathrm{t}}$ and $k_{r}$, respectively. The joint and soil springs are assumed to be bi-linearly elastic and the inertia and damping forces are neglected under the static load assumption. Based on these assumptions, when a ground motion is transmitted to a pipeline directly by soil spring, the governing axial and lateral equations of the pipeline are written by

a) axial direction

$$
-E A \frac{d^{2} u}{d x^{2}}+k_{s x} u=k_{s x} u_{s}
$$

b) lateral direction

$$
E I \frac{d^{4} v}{d x^{4}}+k_{s y} v=k_{s y} v_{s}
$$

where $u, v=$ axial and lateral displacements of the pipeline respectively, $E, A$ and $I=$ Young's modulus, cross sectional area and geometrical moment of inertia of the pipeline respectively, $u_{\mathrm{s}}, v_{\mathrm{s}}=$ axial and lateral displacements of input ground motion respectively, $k_{\mathrm{sx}}, k_{\mathrm{sy}}=$ axial and lateral soil spring constants respectively.

In this study axial deformation of eq. (2) and bending deformation of eq. (3) are uncoupled. For prevention of numerical error accumulation, modified transfer matrix method is adopted. According to this method, the vector $y$ for the displacements and the vector $z$ for the forces are correlated as follows

$$
\begin{aligned}
& \vec{a} y+\vec{b} z=\vec{c} \\
& \bar{a} y+\overleftarrow{b} z=\vec{c}
\end{aligned}
$$


where $a, b$ are square matrices, and the symbol right direction arrow, left direction arrow mean the transfer from left to right and from right to left, respectively. Here the state vector $V$ at the pipeline element is defined as

$$
V=[y, z]
$$

where $y=[u, v, \phi], z=[N, M, Q], \phi, N, M, Q=$ deflection angle, axial force, bending moment and shear force at the end of pipeline segment. Then eqs (2) and (3) are also represented by the system equation as

$$
\frac{d V}{d x}=A V+f(x)
$$

where

$$
A=\left[\begin{array}{cccccc}
0 & 0 & 0 & -1 / E A & 0 & 0 \\
0 & 0 & -1 & 0 & 0 & 0 \\
0 & 0 & 0 & 0 & -1 / E I & 0 \\
-k_{s x} & 0 & 0 & 0 & 0 & 0 \\
0 & 0 & 0 & 0 & 0 & 1 \\
0 & -k_{s y} & 0 & 0 & 0 & 0
\end{array}\right], f(x)=\left[\begin{array}{c}
0 \\
0 \\
q(x) \\
0 \\
0 \\
p(x)
\end{array}\right]
$$

$q(x)=k_{\mathrm{sx}} u_{\mathrm{s}}=$ distributed axial load, $p(x)=k_{\mathrm{sy}} v_{\mathrm{s}}=$ distributed lateral load.

A general solution of eq. (7) is given by

$$
V(x)=e^{A x} V(0)+e^{A x} \int_{0}^{x} e^{-A s} f(s) d s
$$

where $e^{A x}=$ transfer matrix.

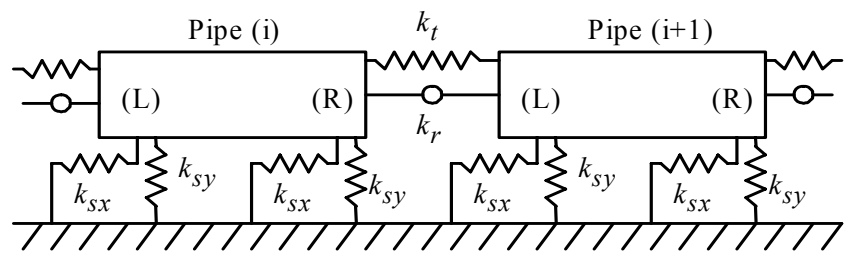

Figure 2: Modeling of pipeline-soil system.

The governing axial and lateral equations (2) and (3) are written as same as eq. (7)

$$
\frac{d}{d x}\left\{\begin{array}{c}
u \\
u^{\prime}
\end{array}\right\}=\left[\begin{array}{cc}
0 & 1 \\
\beta_{1}^{2} & 0
\end{array}\right]\left\{\begin{array}{l}
u \\
u^{\prime}
\end{array}\right\}+\left\{\begin{array}{l}
0 \\
-\beta_{1}^{2} u_{s}
\end{array}\right\}
$$




$$
\frac{d}{d x}\left\{\begin{array}{c}
v \\
v^{\prime} \\
v^{\prime \prime} \\
v^{\prime \prime \prime}
\end{array}\right\}=\left[\begin{array}{cccc}
0 & 1 & 0 & 0 \\
0 & 0 & 1 & 0 \\
0 & 0 & 0 & 1 \\
-4 \beta_{2}^{4} & 0 & 0 & 0
\end{array}\right]\left\{\begin{array}{c}
v \\
v^{\prime} \\
v^{\prime \prime} \\
v^{\prime \prime \prime}
\end{array}\right\}+\left\{\begin{array}{c}
0 \\
0 \\
0 \\
4 \beta_{2}^{4} v_{s}
\end{array}\right\}
$$

where $\beta_{1}=\sqrt{k_{s x} / E A}, \quad \beta_{2}=\sqrt[4]{k_{s x} / E I}$, means the differential $d / d x$.

Using the vector $V$, the field transfer matrix $e^{A x}$ can be obtained. At the joint between the pipe segments $i$ and $i+1$, the following relations are obtained by considering the conditions of displacements and equilibrium

$$
\begin{aligned}
& u_{i+1}^{L}=u_{i}^{R}-N_{i}^{R} / k_{T}, \quad v_{i+1}^{L}=v_{i}^{R}, \quad \phi_{i+1}^{L}=\phi_{i}^{R}-M_{i}^{R} / k_{R}, \\
& N_{i+1}^{L}=N_{i}^{R}, \quad M_{i+1}^{L}=M_{i}^{R}, \quad Q_{i+1}^{L}=Q_{i}^{R}
\end{aligned}
$$

where $k_{T}, k_{R}=$ spring constant of joint for translation and rotation, respectively.

\section{Results and considerations}

Although the numerical computations for the seismic response of surface ground layers are conducted for the Kobe ground models [2], these results and considerations are omitted here for the limitation of the paper space. Only the results and considerations of the responses of pipelines subjected the large ground displacements during huge earthquakes are shown here.

Numerical computations are carried out for the responses of axial and lateral displacements and internal forces of pipelines. The input ground displacement is assumed to be uniformly distributed over the pipeline length. Table 1 shows the examples of the maximum acceleration records observed during the 2011 off the Pacific coast of Tohoku Earthquake. From these values of maximum accelerations, the levels of the maximum values of velocities and displacements may be estimated to be over $0.5-1.0 \mathrm{~m} / \mathrm{s}$ and $\mathrm{m}$, respectively. The reference pipeline dimensions are shown in Table 2. The reference soil spring characteristic is assumed to be represented by bi-linear coefficient of subgrade reaction as shown in Figure 3. The resistant characteristics of soft and hard joint springs are shown in Figures 4 and 5, respectively. These soft and hard joints, which are named GM-type and S-type joint, respectively in Japan, are used as the reference joints for segmented pipelines. The pipeline cases of the both ends fixed type and the cantilever type are evaluated subjected to lateral and longitudinal input ground displacements in Table 3, respectively.

Table 1: $\quad$ Acceleration record data $\left(\mathrm{m} / \mathrm{s}^{2}\right)$.

\begin{tabular}{|c||c|c|c|c|}
\hline Observed & N-S & E-W & U-D & Composed \\
\hline \hline Tsukidate & 27.0 & 12.68 & 18.80 & 29.33 \\
\hline Shiogama & 7.58 & 19.69 & 5.01 & 20.19 \\
\hline Hitachi & 15.98 & 11.86 & 11.66 & 18.45 \\
\hline
\end{tabular}


Table 2: $\quad$ Reference pipe parameters.

\begin{tabular}{|c|c|}
\hline Physical items & Values(unit) \\
\hline Material of segment & Ductile cast iron \\
\hline Nominal diameter & $1100(\mathrm{~mm})$ \\
\hline Thickness & $16.0(\mathrm{~mm})$ \\
\hline Total length & $100(\mathrm{~m})$ \\
\hline Young modulus & $1.57 \times 10^{8}\left(\mathrm{kN} / \mathrm{m}^{2}\right)$ \\
\hline Specific gravity & 7.15 \\
\hline Tensile strength & $3.92 \times 10^{5}\left(\mathrm{kN} / \mathrm{m}^{2}\right)$ \\
\hline Bending strength & $5.59 \times 10^{5}\left(\mathrm{kN} / \mathrm{m}^{2}\right)$ \\
\hline Allowable joint expansion & $50(\mathrm{~mm})$ \\
\hline Allowable joint rot. angle & $5($ degree $)$ \\
\hline
\end{tabular}

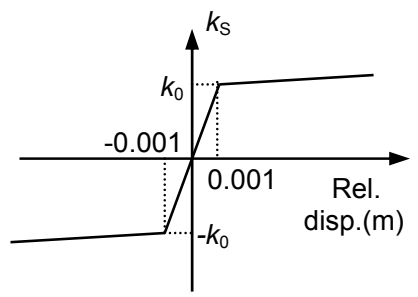

Figure 3: Characteristics of soil spring.

Table 3: $\quad$ Input ground displacements.

\begin{tabular}{|c|c|c|}
\hline Ground condition & Direction & Displacement $(\mathrm{m})$ \\
\hline \hline \multirow{2}{*}{ Normal } & Axial & $0.01-0.05$ \\
& Lateral & $0.1-1.0$ \\
\hline \multirow{2}{*}{ Liquefaction } & Axial & $0.1-0.5$ \\
& Lateral & $1.0-5.0$ \\
\hline
\end{tabular}

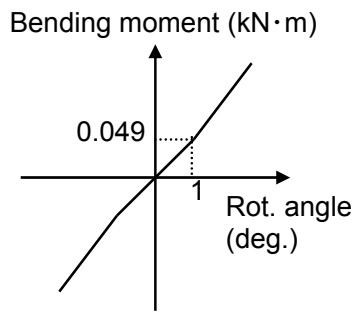

(a) Rotational joint spring

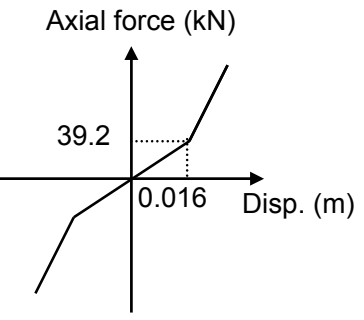

(b) Axial joint spring

Figure 4: Characteristics of soft joint spring (GM-type). 


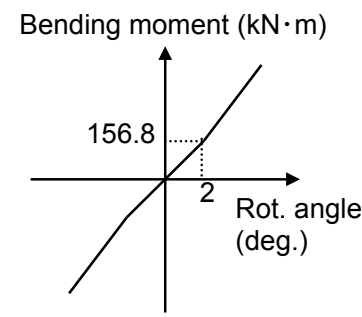

(a) Rotational joint spring

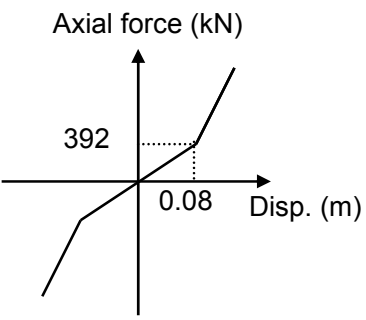

(b) Axial joint spring

Figure 5: Characteristics of hard joint spring (S-type).

Figures 6 and 7 show the distributions of the axial responses of pipeline displacement and axial force, respectively, and (a), (b) in both figures show the cases with soft and hard joint, respectively. Axial input ground displacement $0.01 \mathrm{~m}$ per unit length $1 \mathrm{~m}$ is almost accumulated at the joint as the expansion displacement. Maximum axial force $5100 \mathrm{kN}$ and $6300 \mathrm{kN}$ of soft and hard joints, respectively, which means maximum axial stress about $90,910 \mathrm{kN} / \mathrm{m}^{2}$ $112,300 \mathrm{kN} / \mathrm{m}^{2}$, respectively, are below tensile strength.

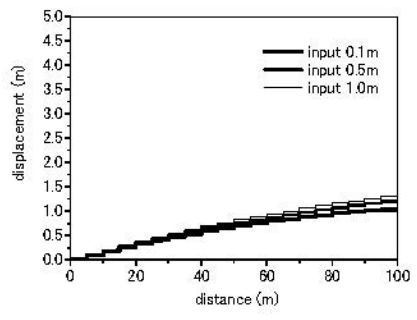

(a) Soft joint

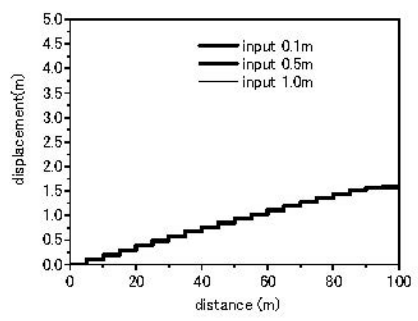

(b) Hard joint

Figure 6: Distribution of axial displacement.

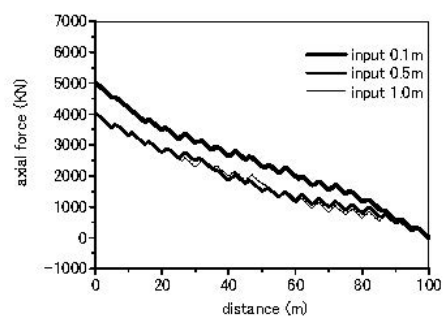

(a) Soft joint

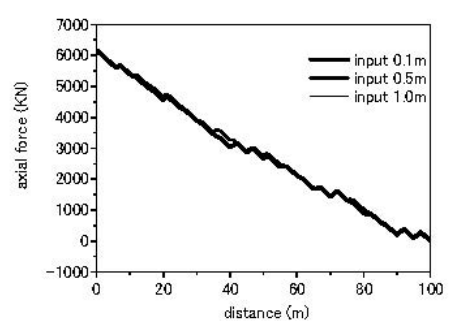

(b) Hard joint

Figure 7: Distribution of axial force.

Figures 8 and 9 show the distribution of the responses of lateral displacement and bending moment of pipelines, respectively, and (a), (b) in both figures show 
the same cases as Figure 6 and 7. Maximum lateral displacement of pipeline is $0.8 \mathrm{~m}$ as for $0.1 \mathrm{~m}$ input of soil. Maximum bending moment $2734 \mathrm{kNm}$ which means bending stress about $190,000 \mathrm{kN} / \mathrm{m}^{2}$ is below tensile strength.

Figures 10 and 11 show the distributions of the responses of joint expansion and joint rotational angle, respectively, and (a), (b) in both figures show the same cases as Figures 6-9. Axial input soil displacements are almost accumulated at the joint as the expansion displacement. Maximum joint expansions are over almost $90.0 \mathrm{~mm}$ and larger than allowable one $50 \mathrm{~mm}$. Maximum joint rotational angle in Figure 11 are over 10 degrees and larger than the allowable value 5 degrees. This means breakage of joint possibly occurs.

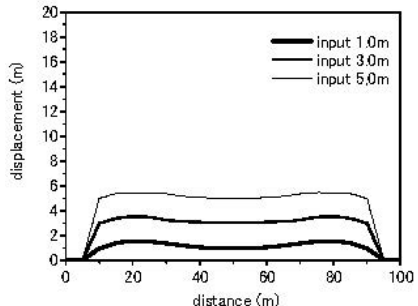

(a) Soft joint

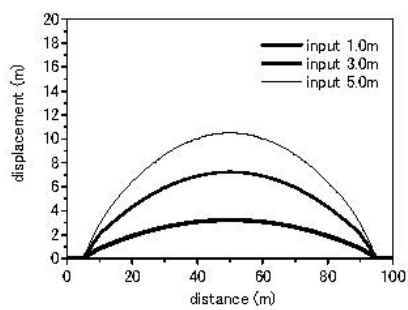

(b) Hard joint

Figure 8: Distribution of lateral displacement.

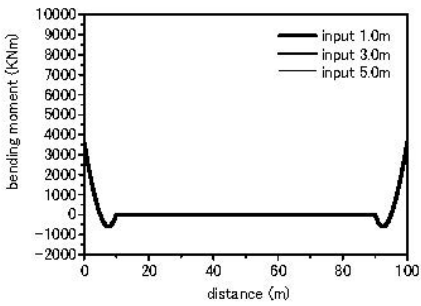

(a) Soft joint

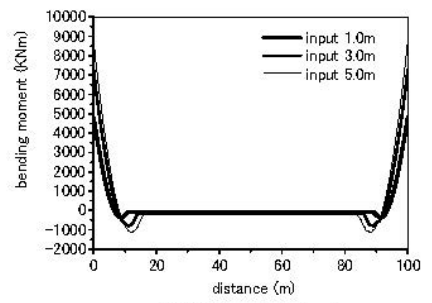

(b) Hard joint

Figure 9: Distribution of bending moment.

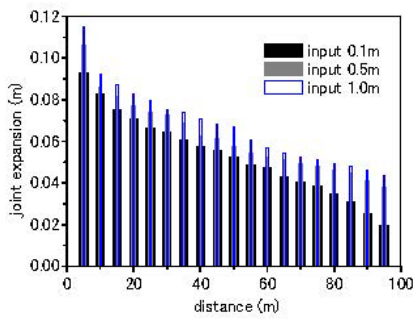

(a) Soft joint

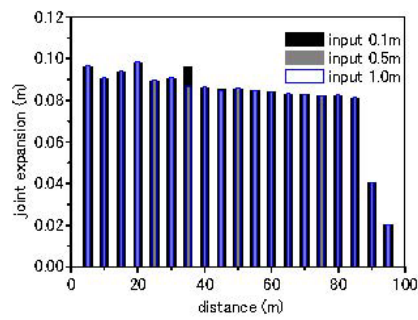

(b) Hard joint

Figure 10: Joint expansion of axial direction. 


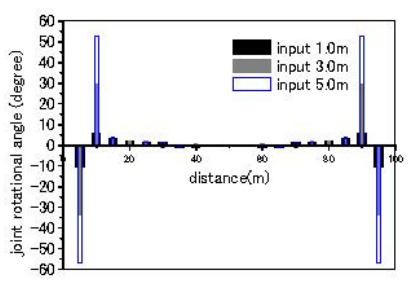

(a) Soft joint

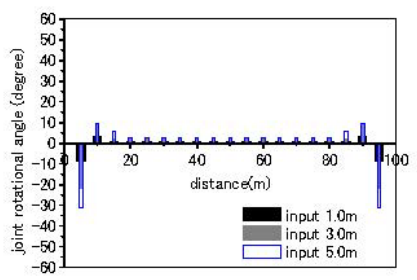

(b) Hard joint

Figure 11: Joint rotational angle.

Figures 12(a) and (b) show the distribution of the responses of lateral displacement and bending moment of pipelines, respectively, for the case of liquefaction ground and with soft joint. Maximum lateral displacement of pipeline reaches to $10.0 \mathrm{~m}$ and maximum bending moment reaches to $1400 \mathrm{kNm}$ for $5.0 \mathrm{~m}$ input of ground. In Figure 12(b) maximum bending stress about $190,000 \mathrm{kN} / \mathrm{m}^{2}$ is still smaller than tensile strength.

Figures 13(a) and (b) show the distributions of responses of joint expansion and joint rotational angle, respectively, in the same case as Figure 12. Maximum joint expansion is slightly over the allowable value $50 \mathrm{~mm}$ only for the ground input 5 meter and maximum joint rotational angle is larger than the 5 degrees for all the ground input level from $1 \mathrm{~m}$ to $5 \mathrm{~m}$. In Figure 13 it means that the joint breakage occurs for the large input of liquefaction ground.

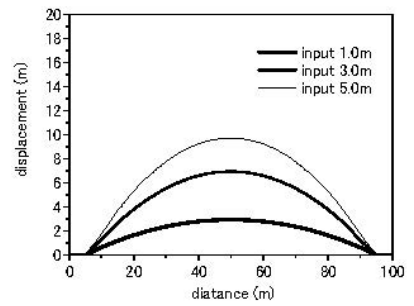

(a) Lateral displacement

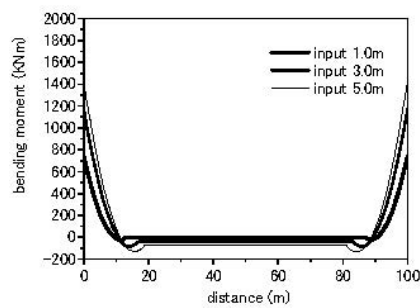

(b) Bending moment

Figure 12: Response of lateral direction input (soft joint, liquefaction ground).

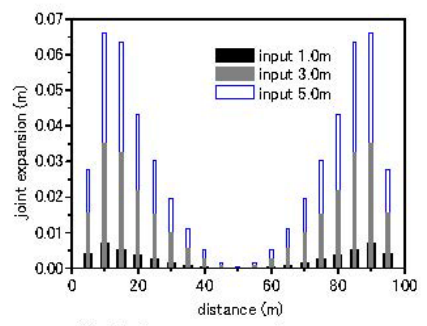

(a) Joint expansion

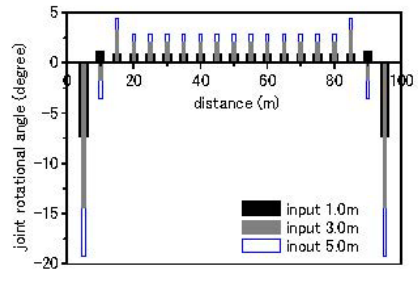

(b) Joint rotational angle

Figure 13: Response of lateral direction input (soft joint, liquefaction ground). 


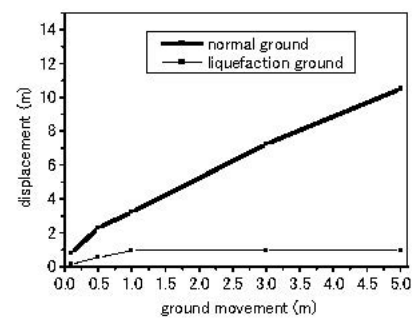

(a) Lateral displacement

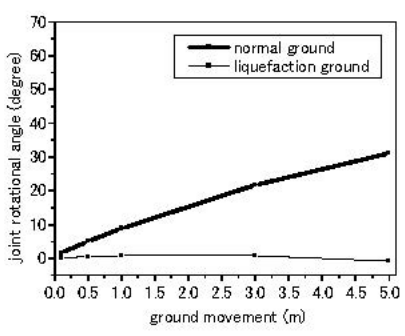

(b) Joint rotational angle

Figure 14: Responses versus lateral input displacements (hard joint).

Figures 14(a) and (b) show the responses of lateral displacement of pipeline and joint rotational angle, respectively, versus input ground movement. In Figure 14 the responses of pipeline and joint increase as increasing of input ground movement in the case of normal ground, but for the liquefaction ground these responses keep almost constant value as input ground movement increases.

\section{Conclusions}

In this study, an evaluating method for seismic responses of pipelines is proposed and response characteristics of pipelines subjected to large ground displacement during huge earthquake are investigated. The method consists of two different kinds of analyses which are the seismic response analysis of surface ground layers and the pseudo static response analysis of pipelines. The numerical computational results obtained for various models with changing conditions of ground and pipelines are investigated. The proposed method is effective on evaluating the situation of whole conditions of pipelines and surface ground layers. From the detailed investigation of the responses of pipeline and joint, breakage of joints and pipeline segments are evaluated and related with various conditions of pipelines, joints and grounds.

\section{References}

[1] Akiyoshi, T., Fuchida, K., et al., A Nonlinear Seismic Response Analysis Method for Saturated Soil-Structure System with Absorbing Boundary, Int. J. Numeric. and Analytic. Methods in Geomech., 20(5), pp. 307-329, 1996.

[2] Akiyoshi, T., Fuchida, K., et al., Local site effects of transient dynamic characteristics of irregularly layered grounds, Proc.4th Int. Conf. on Recent Advan. in Geotech. Earthq. Eng. and Soil Dyn., Paper No. 3. 11, 2001.

[3] Iai, S. et al., Strain space plasticity model for cyclic mobility, Soils and Foundations, JSSMFE, Vol.32, No.2, pp. 1-15, 1992.

[4] Fuchida, K., Wang, L.R.L. \& Akiyoshi, T., Parametric Analysis of Buried Pipelines Subjected to Liquefied Ground Movements, Proc. of 5th U.S. National Conf. on Earthq. Eng., pp. 959-968, 1994. 
[5] Fuchida, K., Akiyoshi, T., et al., Preventive effect of ground improvement on liquefaction and destructive deformation of pipelines, Proc. of Third China-Japan-US Trilateral Symp. on Lifeline Earthq. Eng., pp.141-148, 1998.

[6] Fuchida, K., Evaluation method for behavior of pipelines during earthquakes, Proc. of 7th Int. Conf. on Earthq. Resist. Eng. Struct., pp. 369379, 2009.

[7] Fuchida, K., Evaluation of response characteristics of buried pipelines during earthquakes, Proc. of 8th Int. Conf. on Earthq. Resist. Eng. Struct., pp. 163-172, 2011. 